images at the smallest pressure difference at which the two images could still be resolved was measured and taken to be the half-value width of the line.

Now the half-value width of a line with no fine structure (a simple line) due to the Doppler effect arising from the movement of the atoms is equal to $\nu \times \sqrt{\theta / M} / 1 \cdot 2 \times 10^{8}$, where $\nu$ is the frequency of the line, $M$ is the atomic weight and $\theta$ the absolute temperature of the radiating atoms. In the following table the observed half-value width of the lines examined is compared with the calculated width for a simple line.

\begin{tabular}{|c|c|c|c|c|c|c|c|}
\hline Line & $\begin{array}{c}3280 \\
1 S_{1 / 2}-2^{2} P_{3 / 2}\end{array}$ & $\begin{array}{c}3380 \\
1 S_{1 / \mathbf{s}^{2}-\mathcal{L}^{2} P_{1 / 3}}\end{array}$ & $\begin{array}{c}5209 \\
2^{2} P_{1 / 2}-3^{2} D_{3 / 3} \\
\end{array}$ & $\begin{array}{c}5465 \\
2 P^{2} P^{2}-3^{2} D_{8 / 3} \\
\end{array}$ & $\begin{array}{c}5471 \\
2^{2} P_{\mathbf{3} / 2}-3^{2} D_{\mathrm{s}: \mathbf{3}} \\
\end{array}$ & $\begin{array}{c}4055 \\
2^{2} P_{1 / 2} 4^{2} D_{3 / 2}\end{array}$ & $2^{2} P_{3 / 2}^{421}$ \\
\hline $\begin{array}{l}\text { Ohserved } \\
\text { half-value } \\
\text { willth of line }\end{array}$ & $0.07 \mathrm{~cm}^{-1}$ & $0.07 \mathrm{~cm}^{-1}$ & $0.04 \mathrm{~cm} .^{-1}$ & $0.04 \mathrm{~cm}^{-1}$ & $0.04 \mathrm{~cm}^{-2}$ & $0.05 \mathrm{~cm}^{-1}$ & $0.05 \mathrm{~cm}^{-1}$ \\
\hline $\begin{array}{l}\text { Calculated } \\
\text { width of } \\
\text { simple line }\end{array}$ & $0.07 \mathrm{~cm}^{-1}$ & $0.07 \mathrm{~cm}^{-1}$ & $0.04 \mathrm{~cm}^{-1}$ & $0.04 \mathrm{~cm}^{-1}$ & $0.04 \mathrm{~cm}^{-1}$ & $0.05 \mathrm{~cm}^{-1}$ & $0.05 \mathrm{~cm}^{-1}$ \\
\hline
\end{tabular}

None of the observed lines has a greater width than that calculated for a simple line. It can there. fore be concluded from the observed width of the lines of the diffuse series that any structure in the terms $2^{2} P_{1 / 2}, 2^{2} P_{3 / 2}, 3^{2} D_{3 / 2}$ and $3^{2} D_{5 / 2}$ must be less than $0.04 \mathrm{~cm} .^{-1}$; and from the width of the resonance lines, any structure in the $1 S_{1 / 2}$ term must be less than $0.07 \mathrm{~cm} .^{-1}$ (The triplet structure observed by Mohammad and Sharma ${ }^{4}$ in the resonance lines is due to self-reversal of the lines given by the vacuum arc lamp they used and also a ghost in the Lummer plate fringes in the ultra-violet.) This simplicity of the $1 S_{1 / 2}$ term is of the greatest interest for it signifies that any doubling of this level arising from magnetic moments of the nuclei of the silver isotopes must be smaller than $0.07 \mathrm{~cm}^{-1}$ This seems to indicate an abnormally low magnetic moment of the silver nuclei; for the $1 S_{1 / 2}$ term of copper has a doublet structure with a separation of about $0.3 \mathrm{~cm} .^{-1}$, and since the $1 S_{1 / 2}$ term of silver corresponds to a more penetrating orbit, it would be expected that, if the silver nuclei possessed magnetic moments comparable with those of the copper nuclei, the $1 S_{1 / 2}$ term of silver would possess a doublet separation of the order of $0.5 \mathrm{~cm} .^{-1}$

It has been suggested to me by Dr. S. Tolansky that the absence of structure in the deeply penetrating $1 S_{1 / 2}$ level does not prove that the magnetic moments of the silver nuclei are abnormally small. In the spectrum of thallium, the $2 S_{1 / 2}$ and $2^{2} P_{1 / 2}$ terms possess large hyperfine structures, while the $2^{2} P_{3 / 2}$ term, which corresponds to an almost equally deeply penetrating orbit, possesses no observable structure. No explanation of this anomaly has been found, and it must therefore be considered possible that, owing to a similar cause, a normal magnetic moment of the nuclei gives rise to no observable structure in the $1 S_{1 / 2}$ term of silver. The absence of fine structure could also be explained by ascribing a mechanical moment of 0 to the nuclei of silver; but this seems to be improbable as silver possesses two isotopes of odd atomic weight (107 and 109) and all atoms of odd atomic weight hitherto investigated. have been found to possess mechanical moments.

Clarendon Laboratory, D. A. JAckson. Oxford. April 4.

1 D. A. Jackson, Proc. Roy. Soc., A, 123, 508; 1930.

2 D. A. Tackson, Z. Phys., 75, 229; 1932.

- W. Mohammad and P. N. Sharma, Indian J. Phys., 6, 75 ; 1931.

\section{Fine Structure of the Resonance Ag I Lines}

THE structure of the silver lines $3280 \mathrm{~A}$. and $3383 \mathrm{~A}$. have been examined with Lummer plates by W. Mohammad and P. N. Sharma using a specially constructed vacuum arc. They report identical triplet structures of intensity ratio $5: 5: 1$ and separations of 0.218 and $0.116 \mathrm{~cm}^{-1}$ respectively, the weaker component being on the violet side. They therefore attribute this structure to the common $5 s^{2} S_{\frac{1}{2}}$ term, which, with a $J$ value of $\frac{1}{2}$ should only give a fine structure multiplicity $2 J+1$ when $J \ll I$. A triplet could only arise if there were an isotopic dis. placement effect and provided the $g(I)$ fac. tors of the nucleii dif. fered so that two of the four components superposed. In this somewhat unlikely event the intensity ratios should be very different from the values quoted above since the abundance ratio is unity. This, however, would not be a serious contradiction since it is well known that intensity measurements with resonance lines are necessarily unreliable owing to selfabsorption.

We have examined these lines with 25 and 35 plate reflection echelons, employing water and liquid air cooled hollow cathode discharges. Two hollow cathodes of $8 \mathrm{~mm}$. internal diameter and $15 \mathrm{~cm}$. length were used, the one consisting of 5 per cent silver and 95 per cent cadmium, the other with the proportions reversed.

With both cathodes, the two lines appeared single, of a total width not exceeding $0.050 \mathrm{~cm}^{-1}$, when the discharge current was low $(0.1 \mathrm{amp}$.). As the current was increased these particular lines broadened while the other lines remained equally sharp. With the silver cathode a sharp reversal occurs at about 0.2 amp., the width of the clear centre band steadily increasing with heavier currents until, with a current of $0.8 \mathrm{amp}$., the separation between the wings of the reversed line amounts to $0.13 \mathrm{~cm}^{-1}$. With the cadmium cathode the current could be increased to 0.4 amp. without reversal.

It may be concluded, therefore, that the structure observed by Mohammad and Sharma is a case of self-reversal due to the unsuitability of the vacuum arc source. The total structure width of the lines must be appreciably less than $0.050 \mathrm{~cm}^{-1}$

The peculiar absence of structure in these and other Ag I lines is explained in a forthcoming paper (Proc. Phys. Soc.) by Dr. Tolansky, who infers that it is due to anomalous external electron coupling, and not necessarily to zero spin or small magnetic moment.

Wheatstone Laboratory, W. E. WrLliams.

King's College,

Strand, W.C.2.

March 30.

${ }^{1}$ Indian J. Phys., 6, 75; 1931.

The 'Tail' of the Male American Toad, Ascaphus

Ascaphus (North America) and Liopelma (New Zealand) constitute, according to Dr. G. K. Noble, the Liopelmidæ, the most primitive anuran family in existence. Apart from the importance of Ascaphus from the general phylogenetic aspect, it has often been credited with possessing a tail. Noble has 